\title{
Airborne Observations of Far-Infrared Emission Lines from the Photodissociation Regions in Planetary Nebulae: Properties and Masses of the Neutral Components
}

\author{
Harriet L. Dinerstein ${ }^{1}$, Michael R. Haas \& Edwin F. Erickson ${ }^{2}$ and \\ Michael W. Werner ${ }^{3}$ \\ ${ }^{1}$ University of Texas at Austin; ${ }^{2}$ NASA Ames Research Center; ${ }^{3}$ Jet Propulsion Laboratory
}

Measurements of the main far-infrared cooling lines from photodissociation regions in planetary nebulae were obtained with NASA's Kuiper Airborne Observatory, using a moderate-resolution far-infrared cooled grating spectrometer (Erickson et al. 1985). We surveyed about 20 nebulae in the [O I] $63 \mu \mathrm{m}$ line, and detected it in most of the observed objects. We found that its strength correlates well with the total far-infrared power, with an average ratio of about $0.2 \%$, as predicted by models of photodissociation regions (e.g. Tielens \& Hollenbach 1985; Wolfire et al. 1990). Strong $63 \mu \mathrm{m}$ emission is absent only for objects in which the ionization of the gas is just beginning and for a few evolved objects where the ionized nebula is probably matter-bounded.

For several planetary nebulae we also measured [O I] 145 and [C II] $157 \mu \mathrm{m}$, which together with [O I] $63 \mu \mathrm{m}$ provide diagnostics of temperature and density (e.g. Dinerstein 1995). For the best observed objects, NGC 6572, NGC 7027, BD+30 3639 , and IC 418 , the line ratios are remarkably uniform: $\mathrm{F}(63 \mu \mathrm{m}) / \mathrm{F}(146 \mu \mathrm{m})=20 \pm 5$ and $\mathrm{F}(63 \mu \mathrm{m}) / \mathrm{F}(157$ $\mu \mathrm{m})=8 \pm 2$ (Dinerstein et al. 1995). However, some of these nebulae are known to be carbon-rich. While the observed line ratios taken at face value suggest physical conditions of $\log \mathrm{n}=4.0, \mathrm{~T}=500 \mathrm{~K}$, corrections for the $\mathrm{C} / \mathrm{O}$ ratios measured in the ionized gas move the inferred conditions towards $\log n \geq 4.3, T \geq 1000 K$. Although in this regime the line ratios lose some of their diagnostic power, the upper level of the [C II] line is nearly thermalized so that this line becomes a good mass indicator. We find a surprisingly uniform value for these four nebulae: about 0.1 solar mass. Since this number depends on assumed distance, a more robust conclusion is that the mass of warm photodissociated gas is comparable to the mass of ionized gas in each nebula. Furthermore, since the far-infrared lines arise only from gas at $\mathrm{T} \geq 200-300 K$, the derived mass gives a lower limit to the total mass of the neutral/molecular envelope. We conclude that most bright, compact planetary nebulae are indeed radiation-bounded, as advocated by Pottasch (1980), and that in order to understand the true structure and evolution of planetary nebulae, it will be essential to study their neutral as well as molecular components.

\section{REFERENCES}

Dinerstein, H.L. 1995, in The Analysis of Emission Lines, eds. Williams \& Livio (CUP), 134. Dinerstein, H.L., Haas, M.R., Erickson, E.F., \& Werner, M.W. 1995, in Airborne Astronomy Symposium on the Galactic Ecosystem, eds. M.R. Haas, J.A. Davidson, \& E.F. Erickson, ASP Conf. Ser., Vol. 73, 365.

Erickson, E.F. et al. 1985, Proc. Soc. Photo-Optical Instrum. Engineers, 509, 129.

Pottasch, S.R., 1980, Astron. Astrophys., 89, 336.

Tielens, A.G.G.M., \& Hollenbach, D.J. 1985, Astrophys. Journal, 291, 722.

Wolfire, M.G., Tielens, A.G.G.M., \& Hollenbach, D.J. 1990, Astrophys. Journal, 344, 770. 